\title{
“Supporting Information"
}

(5-Arylfuran-2-ylcarbonyl)guanidines as Cardioprotectives through the Inhibition of $\mathrm{Na}^{+} / \mathrm{H}^{+}$Exchanger Isoform-1 (NHE-1)

Sunkyung Lee, Kyu Yang Yi ${ }^{*}$, Sun Kyung Hwang, Byung Ho Lee, Sung-eun Yoo, Kyunghee Lee ${ }^{\dagger}$

Medicinal Science Division, Korea Research Institute of Chemical Technology, Yuseong-gu,

Daejeon 305-600, Korea, Central Research Center of Yuyu Inc., Kyounggi-do 430-804, Korea

* To whom correspondence should be addressed. Phone: +82-42-860-7143. Fax: +82-42-861-1291. E-mail: kyyi@krict.re.kr

$\dagger$ Yuyu Inc. 
Elemenral Analyses Data

\begin{tabular}{|c|c|c|c|c|c|c|c|c|c|}
\hline \multirow[t]{2}{*}{ compd } & \multirow[t]{2}{*}{ Formula } & \multicolumn{4}{|c|}{ Calculated } & \multicolumn{4}{|c|}{ Found } \\
\hline & & $\mathrm{C}(\%)$ & $\mathrm{H}(\%)$ & $\mathrm{N}(\%)$ & $\mathrm{S}(\%)$ & $\mathrm{C}(\%)$ & $\mathrm{H}(\%)$ & $\mathrm{N}(\%)$ & $\mathrm{S}(\%)$ \\
\hline 47 & $\mathrm{C}_{13} \mathrm{H}_{15} \mathrm{~N}_{3} \mathrm{O}_{5} \mathrm{~S}$ & 47.99 & 4.65 & 12.92 & 9.86 & 47.80 & 4.71 & 12.78 & 9.67 \\
\hline 48 & $\mathrm{C}_{13} \mathrm{H}_{14} \mathrm{FN}_{3} \mathrm{O}_{5} \mathrm{~S}$ & 45.48 & 4.11 & 12.24 & 9.34 & 45.45 & 4.22 & 12.24 & 9.34 \\
\hline 49 & $\mathrm{C}_{12} \mathrm{H}_{10} \mathrm{ClN}_{3} \mathrm{O}_{2}$ & 54.66 & 3.82 & 15.94 & & 54.27 & 3.83 & 15.64 & \\
\hline 50 & $\mathrm{C}_{13} \mathrm{H}_{13} \mathrm{~N}_{3} \mathrm{O}_{3}$ & 60.22 & 5.05 & 16.21 & & 59.85 & 4.96 & 15.89 & \\
\hline 51 & $\mathrm{C}_{14} \mathrm{H}_{17} \mathrm{~N}_{3} \mathrm{O}_{5} \mathrm{~S}$ & 49.55 & 5.05 & 12.38 & 9.45 & 49.51 & 5.10 & 12.25 & 9.08 \\
\hline 52 & $\mathrm{C}_{14} \mathrm{H}_{14} \mathrm{~F}_{3} \mathrm{~N}_{3} \mathrm{O}_{5} \mathrm{~S}$ & 42.75 & 3.59 & 10.68 & 8.15 & 42.85 & 3.73 & 10.48 & 7.96 \\
\hline 53 & $\mathrm{C}_{13} \mathrm{H}_{14} \mathrm{~N}_{4} \mathrm{O}_{7} \mathrm{~S}$ & 42.16 & 3.81 & 15.13 & 8.66 & 41.83 & 3.75 & 15.19 & 8.93 \\
\hline 54 & $\mathrm{C}_{12} \mathrm{H}_{12} \mathrm{~N}_{4} \mathrm{O}_{2}$ & 59.01 & 4.95 & 22.94 & & 58.62 & 5.12 & 22.61 & \\
\hline 55 & $\mathrm{C}_{14} \mathrm{H}_{15} \mathrm{~N}_{3} \mathrm{O}_{2}$ & 65.36 & 5.88 & 16.33 & & 65.01 & 6.10 & 15.95 & \\
\hline 56 & $\mathrm{C}_{14} \mathrm{H}_{15} \mathrm{~N}_{3} \mathrm{O}_{3}$ & 61.53 & 5.53 & 15.38 & & 61.21 & 5.42 & 14.99 & \\
\hline 57 & $\mathrm{C}_{15} \mathrm{H}_{17} \mathrm{~N}_{3} \mathrm{O}_{3}$ & 62.71 & 5.96 & 14.63 & & 62.32 & 5.87 & 14.27 & \\
\hline 58 & $\mathrm{C}_{18} \mathrm{H}_{15} \mathrm{~N}_{3} \mathrm{O}_{3}$ & 67.28 & 4.70 & 13.08 & & 67.27 & 4.78 & 13.13 & \\
\hline 59 & $\mathrm{C}_{12} \mathrm{H}_{10} \mathrm{FN}_{3} \mathrm{O}_{2}$ & 58.30 & 4.08 & 17.00 & & 58.48 & 3.88 & 17.01 & \\
\hline 60 & $\mathrm{C}_{12} \mathrm{H}_{10} \mathrm{ClN}_{3} \mathrm{O}_{2}$ & 54.66 & 3.82 & 15.94 & & 54.83 & 3.70 & 15.63 & \\
\hline 61 & $\mathrm{C}_{14} \mathrm{H}_{17} \mathrm{~N}_{3} \mathrm{O}_{6} \mathrm{~S}$ & 47.32 & 4.82 & 11.82 & 9.02 & 47.27 & 4.83 & 12.14 & 8.80 \\
\hline 62 & $\mathrm{C}_{13} \mathrm{H}_{13} \mathrm{~N}_{3} \mathrm{O}_{2}$ & 64.19 & 5.39 & 17.27 & & 64.27 & 5.36 & 17.33 & \\
\hline 63 & $\mathrm{C}_{14} \mathrm{H}_{14} \mathrm{~F}_{3} \mathrm{~N}_{3} \mathrm{O}_{5} \mathrm{~S}$ & 42.75 & 3.59 & 10.68 & 8.15 & 42.77 & 3.65 & 10.43 & 8.33 \\
\hline 64 & $\mathrm{C}_{12} \mathrm{H}_{10} \mathrm{~N}_{4} \mathrm{O}_{4}$ & 52.56 & 3.68 & 20.43 & & 52.87 & 3.64 & 20.09 & \\
\hline 65 & $\mathrm{C}_{12} \mathrm{H}_{12} \mathrm{~N}_{4} \mathrm{O}_{2}$ & 59.01 & 4.95 & 22.94 & & 59.04 & 5.02 & 22.69 & \\
\hline 66 & $\mathrm{C}_{12} \mathrm{H}_{10} \mathrm{FN}_{3} \mathrm{O}_{2}$ & 58.30 & 4.08 & 17.00 & & 58.48 & 3.88 & 17.01 & \\
\hline 67 & $\mathrm{C}_{13} \mathrm{H}_{14} \mathrm{ClN}_{3} \mathrm{O}_{5} \mathrm{~S}$ & 43.40 & 3.92 & 11.68 & 8.91 & 43.21 & 3.91 & 11.58 & 8.78 \\
\hline 68 & $\mathrm{C}_{14} \mathrm{H}_{17} \mathrm{~N}_{3} \mathrm{O}_{6} \mathrm{~S}$ & 47.32 & 4.82 & 11.82 & 9.02 & 46.97 & 4.72 & 12.20 & 9.37 \\
\hline 69 & $\mathrm{C}_{14} \mathrm{H}_{17} \mathrm{~N}_{3} \mathrm{O}_{5} \mathrm{~S}$ & 49.55 & 5.05 & 12.38 & 9.45 & 49.22 & 4.81 & 12.03 & 9.58 \\
\hline 70 & $\mathrm{C}_{13} \mathrm{H}_{10} \mathrm{~F}_{3} \mathrm{~N}_{3} \mathrm{O}_{2}$ & 52.53 & 3.39 & 14.14 & & 52.47 & 3.37 & 14.02 & \\
\hline
\end{tabular}




\begin{tabular}{|c|c|c|c|c|c|c|c|c|c|}
\hline 71 & $\mathrm{C}_{12} \mathrm{H}_{10} \mathrm{~N}_{4} \mathrm{O}_{4}$ & 52.56 & 3.68 & 20.43 & & 52.67 & 3.46 & 20.31 & \\
\hline 72 & $\mathrm{C}_{12} \mathrm{H}_{12} \mathrm{~N}_{4} \mathrm{O}_{2}$ & 59.01 & 4.95 & 22.94 & & 58.74 & 5.11 & 22.59 & \\
\hline 73 & $\mathrm{C}_{12} \mathrm{H}_{9} \mathrm{~F}_{2} \mathrm{~N}_{3} \mathrm{O}_{2}$ & 54.34 & 3.42 & 15.84 & & 54.40 & 3.53 & 15.71 & \\
\hline 74 & $\mathrm{C}_{12} \mathrm{H}_{9} \mathrm{Cl}_{2} \mathrm{~N}_{3} \mathrm{O}_{2}$ & 48.35 & 3.04 & 14.09 & & 48.32 & 3.19 & 13.82 & \\
\hline 75 & $\mathrm{C}_{14} \mathrm{H}_{15} \mathrm{~N}_{3} \mathrm{O}_{4}$ & 58.13 & 5.23 & 14.53 & & 58.08 & 5.34 & 14.33 & \\
\hline 76 & $\mathrm{C}_{14} \mathrm{H}_{15} \mathrm{~N}_{3} \mathrm{O}_{2}$ & 65.35 & 5.88 & 16.33 & & 64.95 & 5.74 & 15.98 & \\
\hline 77 & $\mathrm{C}_{13} \mathrm{H}_{13} \mathrm{~F}_{2} \mathrm{~N}_{3} \mathrm{O}_{5} \mathrm{~S}$ & 43.21 & 3.63 & 11.63 & 8.87 & 43.33 & 3.48 & 11.53 & 9.01 \\
\hline 78 & $\mathrm{C}_{12} \mathrm{H}_{9} \mathrm{~F}_{2} \mathrm{~N}_{3} \mathrm{O}_{2}$ & 54.34 & 3.42 & 15.84 & & 54.28 & 3.42 & 15.90 & \\
\hline 79 & $\mathrm{C}_{12} \mathrm{H}_{9} \mathrm{Cl}_{2} \mathrm{~N}_{3} \mathrm{O}_{2}$ & 48.35 & 3.04 & 14.09 & & 48.02 & 3.31 & 13.80 & \\
\hline 80 & $\mathrm{C}_{14} \mathrm{H}_{15} \mathrm{~N}_{3} \mathrm{O}_{4}$ & 58.13 & 5.23 & 14.53 & & 57.98 & 5.30 & 14.20 & \\
\hline 81 & $\mathrm{C}_{14} \mathrm{H}_{15} \mathrm{~N}_{3} \mathrm{O}_{2}$ & 65.35 & 5.88 & 16.33 & & 65.38 & 5.68 & 16.20 & \\
\hline 82 & $\mathrm{C}_{13} \mathrm{H}_{12} \mathrm{FN}_{3} \mathrm{O}_{2}$ & 59.77 & 4.63 & 16.08 & & 59.78 & 4.47 & 16.10 & \\
\hline 83 & $\mathrm{C}_{13} \mathrm{H}_{12} \mathrm{FN}_{3} \mathrm{O}_{2}$ & 59.77 & 4.63 & 16.08 & & 59.79 & 4.55 & 15.99 & \\
\hline 84 & $\mathrm{C}_{13} \mathrm{H}_{12} \mathrm{FN}_{3} \mathrm{O}_{3}$ & 56.32 & 4.36 & 15.16 & & 55.98 & 4.75 & 14.83 & \\
\hline 85 & $\mathrm{C}_{13} \mathrm{H}_{12} \mathrm{ClN}_{3} \mathrm{O}_{3}$ & 53.16 & 4.12 & 14.31 & & 52.86 & 4.29 & 13.97 & \\
\hline 86 & $\mathrm{C}_{14} \mathrm{H}_{13} \mathrm{ClFN}_{3} \mathrm{O}_{5} \mathrm{~S}$ & 39.31 & 3.06 & 9.82 & 7.50 & 39.34 & 2.88 & 9.62 & 7.74 \\
\hline 87 & $\mathrm{C}_{13} \mathrm{H}_{12} \mathrm{BrN}_{3} \mathrm{O}_{3}$ & 46.17 & 3.58 & 10.66 & & 46.42 & 3.67 & 10.89 & \\
\hline 88 & $\mathrm{C}_{12} \mathrm{H}_{9} \mathrm{~F}_{2} \mathrm{~N}_{3} \mathrm{O}_{2}$ & 54.34 & 3.42 & 15.84 & & 54.40 & 3.64 & 15.85 & \\
\hline 89 & $\mathrm{C}_{12} \mathrm{H}_{9} \mathrm{ClFN}_{3} \mathrm{O}_{2}$ & 51.17 & 3.22 & 14.92 & & 51.13 & 3.34 & 14.95 & \\
\hline 90 & $\mathrm{C}_{14} \mathrm{H}_{15} \mathrm{~N}_{3} \mathrm{O}_{4}$ & 58.13 & 5.23 & 14.53 & & 57.81 & 5.38 & 14.24 & \\
\hline 91 & $\mathrm{C}_{14} \mathrm{H}_{15} \mathrm{~N}_{3} \mathrm{O}_{2}$ & 65.35 & 5.88 & 16.33 & & 65.08 & 5.70 & 15.99 & \\
\hline 92 & $\mathrm{C}_{12} \mathrm{H}_{9} \mathrm{~F}_{2} \mathrm{~N}_{3} \mathrm{O}_{2}$ & 54.34 & 3.42 & 15.84 & & 54.35 & 3.45 & 15.87 & \\
\hline 93 & $\mathrm{C}_{12} \mathrm{H}_{9} \mathrm{Cl}_{2} \mathrm{~N}_{3} \mathrm{O}_{2}$ & 48.35 & 3.04 & 14.09 & & 47.96 & 3.20 & 13.70 & \\
\hline 94 & $\mathrm{C}_{14} \mathrm{H}_{15} \mathrm{~N}_{3} \mathrm{O}_{2}$ & 65.35 & 5.88 & 16.33 & & 64.99 & 5.91 & 16.09 & \\
\hline 95 & $\mathrm{C}_{13} \mathrm{H}_{14} \mathrm{ClN}_{3} \mathrm{O}_{6} \mathrm{~S}$ & 41.55 & 3.75 & 11.18 & 8.53 & 41.37 & 3.44 & 10.87 & 8.31 \\
\hline 96 & $\mathrm{C}_{14} \mathrm{H}_{14} \mathrm{ClN}_{3} \mathrm{O}_{3}$ & 54.64 & 4.59 & 13.65 & & 53.24 & 4.57 & 13.31 & \\
\hline 97 & $\mathrm{C}_{15} \mathrm{H}_{16} \mathrm{ClN}_{3} \mathrm{O}_{3}$ & 55.99 & 5.01 & 13.06 & & 55.61 & 4.87 & 12.89 & \\
\hline
\end{tabular}

